\title{
Non-Arbitrage and the Fundamental Theorem of Asset Pricing: Summary of Main Results
}

\author{
Freddy Delbaen and Walter Schachermayer
}

\begin{abstract}
The concept of no arbitrage roughly says that it is impossible to make money out of nothing. The mathematical translation of this concept uses martingale theory and stochastic analysis. The paper gives an overview of the results obtained by the authors.
\end{abstract}

\section{Introduction and Notation}

Starting from the economically meaningful assumption that $\left(S_{t}\right)_{t \in \mathbb{R}_{+}}$essentially does not allow arbitrage profits, the fundamental theorem of asset pricing allows the probability $\mathbf{P}$ on the underlying probability space $(\Omega, \mathcal{F}, \mathbf{P})$ to be replaced by an equivalent measure $\mathbf{Q}$ such that the process $S$ becomes a (local) martingale under the new measure. This makes it possible to use the rich machinery of martingale theory. The present summary focuses on the question: "What is the precise meaning of the word essentially?" From a purely mathematical point of view, we remark that the proofs of the theorems below turn out to be surprisingly hard and require heavy machinery from the theory of stochastic processes, from functional analysis and also require some very technical estimates. The results are or will be published elsewhere where we will give references to related work of other authors. We apologise that in this summary no bibliographic references are given.

The $\mathbb{R}^{d}$-valued process $S$, sometimes denoted $\left(S_{t}\right)_{t \in \mathbb{R}_{+}}$, is supposed to satisfy mathematical properties that reflect economically meaningful ideas such as no arbitrage. There should be no trading strategy $H$ for the process $S$, such that the final payoff described by the stochastic integral $(H \cdot S)_{\infty}$, is a nonnegative function, strictly positive with positive probability. The economic interpretation is that by betting on the process $S$ and without bearing any risk, it should not be possible to make something out of nothing.

1991 Mathematics Subject Classification. Primary 60G44; Secondary 46N30, 46E30, 90A09, $60 \mathrm{H} 05$.

Key words and phrases. martingale, equivalent martingale measure, representing measure, risk neutral measure, hedging, stochastic integration, mathematical finance. 
Mathematically a buy-and-hold strategy is described as an integrand of the form $H=f \mathbf{1}_{] T_{1}, T_{2} \rrbracket}$, where $T_{1} \leq T_{2}$ are finite stopping times and $f$ is $\mathcal{F}_{T_{1}}{ }^{-}$ measurable. The advantage of using such integrands is that they have a clear interpretation: when time $T_{1}(\omega)$ comes up, buy $f(\omega)$ units of the financial asset, keep them until time $T_{2}(\omega)$ and sell. The use of stopping times is interpreted as the use of signals coming from available, observable information. This explains why in financial theories the filtration and the derived concepts such as predictable processes, are important. A linear combination of such buy-and-hold strategies is called a simple integrand. Even if the process $S$ is not a semi-martingale, the stochastic integral $(H \cdot S)$ for $H=f \mathbf{1}_{\rrbracket T_{1}, T_{2} \rrbracket}$ can be defined as the process $(H \cdot S)_{t}=\left(S_{\min \left(t, T_{2}\right)}-S_{\min \left(t, T_{1}\right)}\right) f$.

We showed that in the general case simple integrands are not sufficient to characterise these processes that admit an equivalent martingale measure. On the other hand the use of general integrands leads to other problems. The first is that $H \cdot S$ has to exist. The hypothesis that $S$ is a semi-martingale is therefore introduced. Earlier work shows that this property follows from very weak noarbitrage properties. A second difficulty is the problem of doubling strategies ("les martingales" in French). To avoid these pathologies, a lower bound on the losses needs to be introduced. The resulting integrands are called admissible. In the general case, i.e., a time set of the form $[0, \infty[$ or $[0,1]$ and with a possibility of random jumps, the situation is very delicate.

The following notation will be used. The space $(\Omega, \mathcal{F}, \mathbf{P})$ as well as the filtration $\left(\mathcal{F}_{t}\right)_{t \in \mathbb{R}_{+}}$will remain fixed. Economically this means that we are not considering important problems such as inside information or better/faster accessibility to (il)legal information. We suppose that the filtration satisfies the usual assumptions. The space $L^{0}$ denotes the vector space of all real-valued measurable functions defined on $\Omega$, where as usual two functions equal a.s. are identified. Endowed with the topology of convergence in probability, this space becomes a complete, metrisable vector space (i.e. a Fréchet space). The space cannot be given an equivalent norm and there are, in general, no continuous linear functions from $L^{0}$ to $\mathbb{R}$. The space $L^{\infty}$ is the subspace of $L^{0}$ of all bounded functions. Equipped with the obvious norm $\|f\|_{\infty}=\operatorname{ess} \sup |f(\omega)|$, it becomes a Banach space that is the dual of $L^{1}$. The use of separation theorems in the space $L^{\infty}$ poses the problem that the dual space of $L^{\infty}$ is not $L^{1}$ and care has to be taken to work with sets that are weak* ${ }^{*}$ i.e., $\sigma\left(L^{\infty}, L^{1}\right)$, closed. We remark that the two spaces $L^{\infty}$ as well as $L^{0}$ are, among the $L^{p}$ spaces, the only two spaces that remain the same when the original probability measure is replaced by an equivalent one.

$S$ denotes an $\mathbb{R}^{d}$-valued semi-martingale, defined on the filtered probability space $\left(\Omega,\left(\mathcal{F}_{t}\right)_{t \in \mathbb{R}_{+}}, \mathbf{P}\right)$. An $\mathbb{R}^{d}$-valued predictable process $H$ is called $a$-admissible if it is $S$-integrable, if $H_{0}=0$, if the stochastic integral satisfies $H \cdot S \geq-a$ and if $\lim _{t \rightarrow \infty}(H \cdot S)_{t}$ exists a. s. If the integrand $H$ is $a$-admissible for some $a$, then we simply call $H$ admissible. It is understood that vector stochastic integration theory is used. We also need the following sets:

$$
\begin{aligned}
\mathcal{K} & =\left\{(H \cdot S)_{\infty} \mid H \text { is admissible }\right\} \\
\mathcal{K}_{a} & =\left\{(H \cdot S)_{\infty} \mid H \text { is a-admissible }\right\} \\
\mathcal{C}_{0} & =\mathcal{K}-L_{+}^{0} \\
\mathcal{C} & =\mathcal{C}_{0} \cap L^{\infty}
\end{aligned}
$$


The following properties for $S$ all reflect the idea that it is impossible to make something out of nothing. The bar denotes closure taken in the norm topology of $L^{\infty}$. This means that we will have to take care of the particularities related to the duality $\left(L^{1}, L^{\infty}\right)$. We say that the process $S$ satisfies the (NA) or No Arbitrage property if:

$$
\mathcal{K} \cap L_{+}^{0}=\{0\}
$$

which is equivalent to:

$$
\mathcal{C} \cap L_{+}^{\infty}=\{0\} .
$$

The process $S$ is said to satisfy the (NFLVR) property or the No Free Lunch with Vanishing Risk property if:

$$
\overline{\mathcal{C}} \cap L_{+}^{\infty}=\{0\} .
$$

The latter property can be explained as follows. An element $f \in \overline{\mathcal{C}}$ is the limit in $L^{\infty}$-norm of a sequence $\left(f_{n}\right)_{n>1}$ taken in $\mathcal{C}$. If $f \geq 0$ then clearly the sequence of possible losses $\left(f_{n}^{-}\right)_{n \geq 1}$ tends to zero uniformly, i.e., the risk vanishes. The expression "No Free Lunch" is an old expression used already in the early days of the finance literature. Kreps gave the following technical definition of this concept. Let $S$ be a bounded process and let $\mathcal{K}^{\text {simple }}$ be the set of all outcomes with respect to bounded simple integrands. We define $\mathcal{C}^{\text {simple }}$ in the same way,

$$
\mathcal{C}^{\text {simple }}=\left(\mathcal{K}^{\text {simple }}-L_{+}^{0}\right) \cap L^{\infty} .
$$

Kreps says that the càdlàg adapted process satisfies the property of "No Free Lunch," if

$$
\widetilde{\mathcal{C}^{\text {simple }}} \cap L_{+}^{\infty}=\{0\}
$$

where the tilde means the weak* closure. Unfortunately the weak* closure cannot be obtained by sequences. One has to use general concepts such as nets, generalised sequences and/or filters. The economic interpretation of these objects is unclear. It may happen that an element in the weak* closure can only be obtained by an unbounded generalised sequence. On the other hand the very strong requirement of (NFL) implies that $S$ is a semi-martingale and that there is an equivalent martingale measure for the process $S$. The property (NFLVR) is a slightly stronger version than the no-arbitrage condition. However we pay a price (there is no free lunch as you know). We have to introduce the set of outcomes with respect to general admissible integrands. The construction of the stochastic integral has a lot of stability built in. Therefore we can do it with an assumption such as (NFLVR). The exploitation of this stability requires however the use of non-trivial arguments.

The following theorem relates the definition of (NFLVR) to a boundedness property in $L^{0}$.

THEOREM 1. The process $S$ satisfies the property (NFLVR) if and only if it satisfies

1. (NA) and

2. $\mathcal{K}_{1}$ is bounded in the space $L^{0}$. 
We remark that the boundedness of the set $\mathcal{K}_{1}$ has a direct economic interpretation. For outcomes that have a maximal loss bounded by 1 , the profit is bounded in probability, this means that the probability of making a big profit can be estimated from above, uniformly over all such outcomes.

The following theorem gives a way to control the boundedness of the set $\mathcal{K}_{1}$.

THEOREM 2. If there is a positive local martingale $L$ such that $L_{0}=1$ and $L_{\infty}>0$ a.s. as well as a strictly positive real-valued predictable process $\phi$ such that $(\phi \cdot S) L$ is a local martingale, then the set $\mathcal{K}_{1}$ is bounded in $L^{0}$.

The multiplication with a function $\phi$ is done in order to take care of processes with big jumps. We will give more information on this in the last section. We remark that when $\phi$ is a bounded, nonzero real-valued predictable process, then the set of stochastic integrals with respect to $S$ is the same as the set of stochastic integrals with respect to $\phi \cdot S$. So as long as only the stochastic integrals matter, we can always replace $S$ by a "better" process $\phi \cdot S$. For locally bounded processes we usually can take $\phi=1$. We also emphasize that in the above theorem we only require $L$ to be a local martingale. See also the remark after Theorem 6 .

\section{The locally bounded case}

The next theorem shows that, at least for locally bounded semi-martingales, the (NFLVR) property is equivalent to the existence of an equivalent probability measure that turns the price process $S$ into a local martingale. It is easily seen that we may without loss of generality suppose that the process $S$ is bounded, instead of locally bounded. Indeed if $\left(T_{n}\right)_{n \geq 0}$ is an increasing sequence of stopping times such that $T_{0}=0, \lim _{n \rightarrow \infty} T_{n}=\infty$ and such that every stopped processes $S^{T_{n}}$ is bounded, by say $k_{n}$, then we can replace the process $S$ by the process

$$
S^{\prime}=\sum_{n \geq 1} \mathbf{1}_{\rrbracket T_{n-1}, T_{n} \rrbracket} \frac{1}{k_{n} 2^{n}}\left(S^{T_{n}}-S^{T_{n-1}}\right)=\left(\sum_{n \geq 1} \frac{1}{k_{n} 2^{n}} \mathbf{1}_{\left.\rrbracket T_{n-1}, T_{n}\right]}\right) \cdot S .
$$

Showing that there is an equivalent probability such that $S$ becomes a local martingale is then the same as showing that there is a probability that turns $S^{\prime}$ into a martingale.

THEOREM 3. The locally bounded semi-martingale $S$ satisfies the (NFLVR) property if and only if there is an equivalent measure $\mathbf{Q}$ under which $S$ becomes a local martingale. In this case the set $\mathcal{C}$ is closed in the weak* topology $\sigma\left(L^{\infty}, L^{1}\right)$.

The key point is of course to show that the set $\mathcal{C}$ is weak* closed. Because of the (NA) property, the set $\mathcal{C}$ does not intersect the positive cone, and we can separate with a hyperplane given by a function in $L^{1}$. More precisely there is a strictly positive function $g$ in $L^{1}$ such that for all $f \in \mathcal{C}$ we have $\mathbf{E}[f g] \leq 0$. This implies that for elements of the form $f=\mathbf{1}_{A}\left(S_{t}-S_{s}\right)$ where $s<t$ and $A \in \mathcal{F}_{s}$, we necessarily have $\mathbf{E}[f g]=0$, since indeed both $f$ and $-f$ are in $\mathcal{C}$. It is here that we use that the process $S$ is bounded. Normalising $g$ then gives us an equivalent probability $d \mathbf{Q}=g d \mathbf{P}$ that turns $S$ into a martingale. The fact that $\mathcal{C}$ is weak* closed can be proved for general (i. e., not necessarily locally bounded) semi-martingales. However the last step of the proof, both $f$ and $-f$ being in $\mathcal{C}$, fails! The proof of the weak* closure is based upon the following lemma. We first need a definition: 
Definition 4. An element $f \in \mathcal{K}$ is called maximal if for $g \geq f$ a.s. and $g \in \mathcal{K}$ we necessarily have that $f=g$.

It is easily seen that the (NA) property says that 0 is maximal. Also there are no maximal elements if the (NA) property is violated. Therefore we have that $f \in \mathcal{K}_{1}$ is maximal in $\mathcal{K}_{1}$ if and only if it is maximal in $\mathcal{K}$.

Lemma 5. Suppose that the process $S$ satisfies the (NFLVR) property. If $\left(f_{n}\right)_{n \geq 1}$ is a sequence in $\mathcal{K}_{1}$, then there exists a sequence of convex combinations $g_{n} \in \operatorname{convex}\left\{f_{n}, f_{n+1}, \ldots\right\}$ that converges in probability to some function $g: \Omega \rightarrow \mathbb{R}$. Moreover there is a maximal element $f \in \mathcal{K}_{1}$ such that $g \leq f$, i.e., $g \in \mathcal{C}$.

From this lemma the weak* closure is easily deduced. One uses the property that a convex set in $L^{\infty}$ is weak ${ }^{*}$ closed if and only if the intersections with the balls of $L^{\infty}$ are closed for the convergence in probability. We will not give a sketch of the proof of the lemma.

For continuous processes we can do a little bit better as the following theorem shows.

Theorem 6. Suppose that $S$ is continuous with Doob-Meyer decomposition $S=M+A$. If $S$ satisfies the (NA) property, then the following assertions hold:

1. The measure $d A$ is for almost all $\omega \in \Omega$ absolutely continuous with respect to the matrix valued measure $d\langle M, M\rangle$.

2. The predictable density $h$ defined as $d A=h d\langle M, M\rangle$ satisfies the property

$$
T=\inf \left\{t>0 \mid \int_{0}^{t} h_{u} d\langle M, M\rangle_{u} h_{u}=+\infty\right\}>0 \quad \text { a.s. }
$$

3. The exponential local martingale

$$
L_{t}=\exp \left(-\int_{0}^{t} h_{u} d M_{u}-\frac{1}{2} \int_{0}^{t} h_{u} d\langle M, M\rangle_{u} h_{u}\right) \quad \text { is defined. }
$$

4. There is $\mathbf{Q}$, a probability measure absolutely continuous with respect to $\mathbf{P}$, under which $S$ becomes a local martingale and for which $\{d \mathbf{Q} / d \mathbf{P}>0\}=$ $\left\{L_{\infty}>0\right\}$.

5. If $\mathbf{Q}$ is an absolutely continuous probability measure under which $S$ becomes a local martingale, then necessarily $\{d \mathbf{Q} / d \mathbf{P}>0\} \subset\left\{L_{\infty}>0\right\}$.

If $L$ satisfies the property that $L_{\infty}>0$ a.s., then we have a situation that is described by Theorem 2 and hence the set $\mathcal{K}_{1}$ is bounded in $L^{0}$. Indeed by using Itô's calculus and the fact that $S$ and $L$ are continuous, it is easily verified that $L S$ is a local martingale. Together with the (NA) property this implies the (NFLVR) property and the theorem is proved. The case where $L$ can become zero is more difficult to treat.

We remark that, except in the case of complete markets, the change of measure is not necessarily given by the local martingale $L$. There are examples of a strict local martingale $Z$ such that there is an equivalent measure under which $Z$ becomes a uniformly integrable martingale. This means that if we define $S=1 / Z$ and apply the construction above, we find $L=Z$ and hence $L S=1$. However the process $L$ does not define a new probability measure since $\mathbf{E}\left[L_{\infty}\right]<1$. This also shows that for continuous processes $S$ there is a strict separation between the (NA) property and the boundedness of $\mathcal{K}_{1}$. As an illustration of this loose statement, we take, 
on the time interval $[0,1]$, the Bessel process $S$ in three dimensions, $S_{0}=1$ and with its natural filtration. It is easily seen that in this case the only candidate for a density is the strict local martingale $L=1 / S$. We conclude that the process $S$ cannot have an equivalent local martingale measure and hence it cannot satisfy the (NA) property.

This example also shows that when we use $L$ as a description of a foreign currency, there is no way of making arbitrage by betting on this currency. However the people living in the foreign country are faced with an exchange rate given by $S$ and they have a possibility to make winning bets (also called investments)! Of course this paradoxical situation arises from the notion of admissible integrands. This notion is not invariant for currency changes. What is possible for the foreigners is not possible for the domestics! It also shows that a change of numéraire should be done with some extra care.

For the following theorems we need some extra notation. It is assumed that the bounded process $S$ admits an equivalent local martingale measure. Define

and

$$
\mathbf{M}^{\mathrm{e}}(\mathbf{P})=\left\{\begin{array}{l|l}
\mathbf{Q} & \begin{array}{l}
\mathbf{Q} \text { is equivalent to } \mathbf{P} \\
\text { and the process } S \text { is a } \mathbf{Q} \text {-local martingale }
\end{array}
\end{array}\right\}
$$

$$
\mathbf{M}(\mathbf{P})=\left\{\begin{array}{l|l}
\mathbf{Q} & \begin{array}{l}
\mathbf{Q} \text { is absolutely continuous with respect to } \mathbf{P} \\
\text { and the process } S \text { is a } \mathbf{Q} \text {-local martingale }
\end{array}
\end{array}\right\}
$$

Because the process $S$ is assumed to be bounded (or locally bounded), we can easily show that the set $\mathbf{M}(\mathbf{P})$ is the closure in $L^{1}$ of the set $\mathbf{M}^{\mathrm{e}}(\mathbf{P})$. Again for non locally bounded processes this is false as an easy two period example, where $\mathbf{M}(\mathbf{P})$ is not closed, shows.

The weak* closedness of $\mathcal{C}$, the Hahn-Banach theorem, as well as the basic lemma yield:

THEOREM 7. If $f \geq 0$, then

$$
\sup \left\{\mathbf{E}_{\mathbf{Q}}[f] \mid \mathbf{Q} \in \mathbf{M}^{\mathrm{e}}(\mathbf{P})\right\}=\inf \{\alpha \mid \text { There is } g \in \mathcal{K} \text { with } f \leq \alpha+g\} \text {. }
$$

Moreover when the expression is finite, the infimum is a minimum and $g$ can be chosen to be a maximal element.

Using this equality and the characterisation of maximal elements given below, we can give an improvement of a result of Ansel-Stricker and Jacka. The theorem also says when a change of numéraire will not produce unwanted arbitrage opportunities.

THEOREM 8. If $f=(H \cdot S)_{\infty} \in \mathcal{K}$, for $H$ admissible, then are equivalent:

1. $f$ is a maximal element in $\mathcal{K}$.

2. $f$ is a maximal element in $\mathcal{K}_{\|f-\|_{\infty}}$.

3. There is an element $\mathbf{Q} \in \mathbf{M}^{\mathrm{e}}(\mathbf{P})$ for which the process $(H \cdot S)$ is a $\mathbf{Q}$-uniformly integrable martingale.

4. There is an element $\mathbf{Q} \in \mathbf{M}^{\mathrm{e}}(\mathbf{P})$ for which $\mathbf{E}_{\mathbf{Q}}[f]=0$.

THEOREM 9. If $f \geq 0$, then the following assertions are equivalent:

1. There is $\mathbf{Q} \in \mathbf{M}^{\mathrm{e}}(\mathbf{P})$ such that $\mathbf{E}_{\mathbf{Q}}[f]=\sup \left\{\mathbf{E}_{\mathbf{R}}[f] \mid \mathbf{R} \in \mathbf{M}^{\mathrm{e}}(\mathbf{P})\right\}$,

2. $f=\alpha+g$, where $g$ is maximal in $\mathcal{K}$.

3. $f=\alpha+(H \cdot S)_{\infty}$ and $H \cdot S$ is a uniformly integrable martingale for some element $\mathbf{Q} \in \mathbf{M}^{\mathrm{e}}(\mathbf{P})$. 
If $V=\alpha+H \cdot S, H$ admissible and $f=V_{\infty}>0$ a.s., then the above are also equivalent to:

4. $(S / V, 1 / V)$ satisfies the (NA) property.

5. $(S / V, 1 / V)$ has an equivalent local martingale measure.

Both these theorems are proved together and our proof does not use the $H^{1}$ $B M O$ duality theory. Using the Bishop-Phelps theorem from functional analysis we obtain as a corollary:

Theorem 10. If $\mathbf{M}^{\mathrm{e}}(\mathbf{P})=\mathbf{M}(\mathbf{P}) \neq \varnothing$, then $\mathbf{M}^{\mathrm{e}}(\mathbf{P})$ contains exactly one element.

Because of the importance of the maximal elements, it is interesting to investigate the set $\mathcal{K}_{1}^{\max }$ of all these maximal elements.

THEOREM 11. The set $\mathcal{K}_{1}^{\max }$ of maximal elements in $\mathcal{K}$ is a convex cone that is stable for countable convex combinations.

Corollary 12. If $\left(f_{i}\right)_{i \geq 1}$ is a countable family of maximal elements in $\mathcal{K}$, then there is an equivalent local martingale measure $\mathbf{Q}$ for $S$ such that for all $i \geq 1$ we have $\mathbf{E}_{\mathbf{Q}}\left[f_{i}\right]=0$. There are admissible strategies $H^{i}$, generating $f_{i}$, such that the processes $H^{i} \cdot S$ are uniformly integrable martingales under the measure $\mathbf{Q}$.

It is a naive idea to think that for maximal elements $f$ and for every element $\mathbf{Q} \in \mathbf{M}^{\mathrm{e}}(\mathbf{P})$ we always have $\mathbf{E}_{\mathbf{Q}}[f]=0$. The are examples where $\mathbf{E}_{\mathbf{Q}}[f]=0$ for a well chosen measure $\mathbf{Q}$, but where $\mathbf{E}_{\mathbf{R}}[f]<0$ for an even better chosen element $\mathbf{R} \in \mathbf{M}^{\mathrm{e}}(\mathbf{P})$. Moreover we can show that in incomplete markets with continuous prices, such a situation is more the rule than the exception! However one can show that for a maximal element $f$ the set $\left\{\mathbf{R} \mid \mathbf{R} \in \mathbf{M}(\mathbf{P}) ; \mathbf{E}_{\mathbf{R}}[f]=0\right\}$ is a dense $G_{\delta^{-}}$set in $\mathbf{M}(\mathbf{P})$.

Given a convex cone, it is always a good idea to have a look at the vector space generated by it. So let us define

$$
\mathcal{G}=\mathcal{K}^{\max }-\mathcal{K}^{\max }
$$

For an element $g \in \mathcal{G}$ we define the norm

$$
\|g\|_{\mathcal{G}}=\inf \left\{a>0 \mid \text { There exist } f, h \in \mathcal{K}_{a} \text { with } g=h-f\right\} .
$$

It is easy to see that it really defines a norm and that because of the properties of the cone $\mathcal{K}^{\text {max }}$, the space $\mathcal{G}$ is complete for this norm.

THEOREM 13. The space $\mathcal{G}$ with the above norm $\|\cdot\|_{\mathcal{G}}$ becomes a Banach space. We also have that

$$
2\|g\|_{\mathcal{G}}=\sup \left\{\|g\|_{L^{1}(\mathbf{Q})} \mid \mathbf{Q} \in \mathbf{M}^{\mathrm{e}}(\mathbf{P})\right\}, \quad g \in \mathcal{G} .
$$

Although the space $\mathcal{G}$ and its norm are defined in a natural way, we leave it as a challenge to give an economic interpretation of these results.

We end this section with a theorem that says that under a weak form of the (NFLVR) property, the process $S$ is a semi-martingale. The theorem below therefore shows that in finance, the use of processes such as fractional Brownian motion, is not always appropriate. 
THEOREM 14. If for a bounded (or more generally a locally bounded) process $S$,

$$
\overline{\mathcal{C}^{\text {simple }}} \cap L^{\infty}=\{0\},
$$

then $S$ is a semi-martingale. (Again the bar denotes the norm closure in $L^{\infty}$.)

\section{The case of unbounded processes}

The preceding section dealt with locally bounded processes. From a general viewpoint this is not satisfactory. Insurance models typically treat the case of unbounded claims that happen at totally inaccessible stopping times. Also from a mathematical viewpoint it would be nicer to have a result also for the case of unbounded jumps. The need is even greater if one compares the fundamental theorem with the Dalang-Morton-Willinger theorem. This theorem states that for processes indexed by a finite time-set, the no-arbitrage property implies the existence of an equivalent martingale measure. We remark that in the case of the Dalang-Morton-Willinger theorem, the no-arbitrage condition is written without any restriction to admissibility. More precisely they proved the following theorem.

THEOREM 15. Let $\left(S_{n}\right)_{n=0,1, \ldots, N}$ be a d-dimensional process adapted to the filtration $\left(\mathcal{F}_{n}\right)_{n=0,1, \ldots, N}$. Let $\mathcal{K}^{\mathrm{g}}$ be defined as

$$
\mathcal{K}^{\mathrm{g}}=\left\{\sum_{n=1}^{N} f_{n-1}\left(S_{n}-S_{n-1}\right) \mid \text { Each } f_{n}: \Omega \rightarrow \mathbb{R}^{d} \text { is } \mathcal{F}_{n} \text {-measurable }\right\} .
$$

If $\mathcal{K}^{\mathrm{g}} \cap L_{+}^{0}=\{0\}$, then there is an equivalent measure $\mathbf{Q}$ such that the process $S$ is a $\mathbf{Q}$-martingale.

In the general case it turns out that there is no hope to prove the existence of an equivalent (local) martingale measure. We need a more general concept. We say that a semi-martingale $X$ is a sigma-martingale if there is a strictly positive predictable process $\phi$ such that $\phi$ is $X$-integrable and such that $\phi \cdot X$ is a local martingale. In this case an easy - although not trivial - exercise shows that we can require that $\phi$ is bounded and that $\phi \cdot X$ is an $\mathcal{H}^{1}$-martingale. It is not difficult to see that a local martingale is a sigma-martingale and that a sigma-martingale is a local martingale if and only if the process $\phi$ can be taken to be decreasing. The concept of a sigma-martingale was introduced by Emery and Chou who called it "processus de la classe $\Sigma_{m}$." It is related to martingales in the same way as sigma-finite measures are related to finite measures.

Another easy exercise is to show that a sigma-martingale on a finite discrete time filtration (the case of the Dalang-Morton-Willinger theorem), is already a martingale.

We also remark that the set of all stochastic integrals with respect to a $d$ dimensional semi-martingale $S$ is the same as the set of all stochastic integrals with respect to a process of the form $\phi \cdot S$. Hence, for applications in mathematical finance, the concept of sigma-martingales is, in most cases, as good as the more restrictive concept of local martingales.

We can now state the fundamental theorem in its most general form.

THEOREM 16. Let $S=\left(S_{t}\right)_{t \in \mathbb{R}_{+}}$be an $\mathbb{R}^{d}$-valued semi-martingale defined on the stochastic base $\left(\Omega, \mathcal{F},\left(\mathcal{F}_{t}\right)_{t \in \mathbb{R}_{+}}, \mathbf{P}\right)$. Then $S$ satisfies the condition of (NFLVR) 
if and only if there exists a probability measure $\mathbf{Q} \sim \mathbf{P}$ such that $S$ is a sigmamartingale with respect to $\mathbf{Q}$.

The proof of this theorem starts in the same way as the proof in the locally bounded case. One first shows that the set $\mathcal{C}$ is weak* closed. But in this case such a statement can be trivial, since the set $\mathcal{K}$ of outcomes of admissible integrands can be reduced to the set $\{0\}$ ! Once the closedness is proved, we continue with the following result, interesting in itself.

THEOREM 17. With the notation introduced above, let $\mathbf{Q}$ be an equivalent probability measure such that $\mathbf{E}_{\mathbf{Q}}[f] \leq 0$ for every element $f \in \mathcal{C}$. Then for each $\varepsilon>0$ there is an equivalent probability measure $\mathbf{Q}_{0}$ such that $\left\|\mathbf{Q}_{0}-\mathbf{Q}\right\|<\varepsilon$ and such that $S$ is a sigma-martingale for the measure $\mathbf{Q}_{0}$.

We remark that this theorem implies the Dalang-Morton-Willinger theorem and is in fact a little bit more general since we give somewhat more information about the distortion of the measure.

In the rest of this section we suppose that there is a sigma-martingale measure for the process $S$. Let us redefine:

$$
\mathbf{M}^{\mathrm{e}}(\mathbf{P})=\left\{\begin{array}{l|l}
\mathbf{Q} & \begin{array}{l}
\mathbf{Q} \text { is equivalent to } \mathbf{P} \\
\text { and the process } S \text { is a } \mathbf{Q} \text {-sigma-martingale }
\end{array}
\end{array}\right\} .
$$

As is easily seen, for locally bounded processes this set coincides with the set of equivalent local martingale measures.

The results on maximal elements shown above as well as the duality results can be restated also in the case of unbounded processes. But as observed above, the concept of admissible integrands is too restrictive. In order to solve this problem we introduce the concept of $w$-admissible integrands, where $w \geq 1$ is a weight function. The idea is to say that an integrand $H$ is $w$-admissible if $H \cdot S \geq-w$. Here one has to be careful. If $w$ is too small, then there might be no $w$-admissible integrands, this can be the case if we take e.g. $w=1$ (the classical concept of admissibility) and if big jumps are present. If on the other hand we take $w$ too big, then we might be able to use doubling strategies and there will be arbitrage opportunities. The good balance is to use weight functions $w \geq 1$ that satisfy the following properties:

1. There is a strictly positive predictable $S$-integrable function $\varphi$ such that the maximal function of the vector-valued process $\varphi \cdot S$ satisfies $(\varphi \cdot S)^{*} \leq w$. In this case we are sure that there will be enough $w$-admissible integrands.

2. There is a sigma martingale measure $\mathbf{Q} \in \mathbf{M}^{\mathrm{e}}(\mathbf{P})$ such that $\mathbf{E}_{\mathbf{Q}}[w]<\infty$. This will restrict the concept of $w$-admissibility and will prevent the use of doubling strategies.

It is clear that if $\mathbf{M}^{\mathrm{e}}(\mathbf{P})$ is nonempty (as is the case here), then there are weight functions that satisfy both assumptions. We are now ready to define the concept of $w$-admissible integrands.

DEFinition 18. If $w \geq 1$ is a weight function that satisfies both assumptions above, then we say that an $S$-integrable predictable $\mathbb{R}^{d}$-valued process $H$ is $w$ admissible if for each $\mathbf{Q} \in \mathbf{M}^{\mathrm{e}}(\mathbf{P})$ we have

$$
(H \cdot S)_{t} \geq-\mathbf{E}_{\mathbf{Q}}\left[w \mid \mathcal{F}_{t}\right], \quad t \geq 0 .
$$


REMARK 19. We can show that in the preceding definition the requirement

$$
(H \cdot S)_{t} \geq-\mathbf{E}_{\mathbf{Q}}\left[w \mid \mathcal{F}_{t}\right], \quad t \geq 0,
$$

can be restricted to those elements $\mathbf{Q} \in \mathbf{M}^{\mathrm{e}}(\mathbf{P})$ satisfying $\mathbf{E}_{\mathbf{Q}}[w]<\infty$. This yields an equivalent definition.

Let us also define

$$
\mathcal{K}_{w}=\left\{(H \cdot S)_{\infty} \mid(H \cdot S) \geq-n w \text { for some } n \geq 0\right\} .
$$

It is easily seen that the limit exists. Indeed, if $\mathbf{E}_{\mathbf{Q}}[w]<\infty$, then the process $(H \cdot S)_{t}+\mathbf{E}_{\mathbf{Q}}\left[n w \mid \mathcal{F}_{t}\right], t \geq 0$, is a positive supermartingale for the measure $\mathbf{Q}$.

With the set of admissible outcomes we can construct the set of dominated elements:

$$
\mathcal{C}_{w}=\mathcal{K}_{w}-L_{+}^{0}
$$

The following theorem is the equivalent of the result that says that $\mathcal{C}$ is weak* closed.

THEOREM 20. The set $\left(\frac{1}{w} \mathcal{C}_{w}\right) \cap L^{\infty}$ is weak $k^{*}$ closed in $L^{\infty}$.

THEOREM 21. The element $f \in \mathcal{K}_{w}$ is maximal if and only if there is an element $\mathbf{R} \in \mathbf{M}^{\mathrm{e}}(\mathbf{P})$ such that $w \in L^{1}(\mathbf{R})$ and $\mathbf{E}_{\mathbf{R}}[f]=0$.

THEOREM 22. If $f \geq-w$, then we have

$$
\begin{aligned}
\sup \left\{\mathbf{E}_{\mathbf{R}^{\prime}}[f] \mid \mathbf{E}_{\mathbf{R}^{\prime}}[w]<\infty, \mathbf{R}^{\prime}\right. & \left.\in \mathbf{M}^{\mathrm{e}}(\mathbf{P})\right\} \\
& =\inf \left\{\alpha \geq 0 \mid \text { There is } g \in \mathcal{K}_{w} \text { with } f \leq \alpha+g\right\} .
\end{aligned}
$$

The proofs of these results are a combination of the proofs for the locally bounded case together with compactnes results of bounded sequences in the space $\mathcal{H}^{1}$. We remark that even in the case of locally bounded price processes $S$, the above results are more precise.

\section{References}

[DS94a] F. Delbaen and W. Schachermayer, A general version of the fundamental theorem of asset pricing, Math. Ann. 300 (1994), 463-520.

[DS94b] _ Arbitrage and free lunch with bounded risk for unbounded continuous processes, Math. Finance 4 (1994), 343-348.

[DS95a] _ Arbitrage possibilities in Bessel processes and their relations to local martingales, Probab. Theor. Relat. Fields 102 (1995), 357-366.

[DS95b] — The no-arbitrage property under a change of numéraire, Stochastics 53 (1995), $213-226$.

[DS95c] - The no arbitrage property for continuous processes, Ann. of Appl. Probab. 5 (1995), 924-945.

[DS96a] _ , The fundamental theorem of asset pricing for unbounded stochastic processes, preprint (1996).

[DS96b] _ A compactness principle for bounded sequences of $\mathcal{H}^{1}$ martingales, preprint (1996).

[DS97a] _ A simple counter-example to several problems in the theory of asset pricing, which arises generically in incomplete markets, Math. Finance (1997).

[DS97b] _ The Banach space of workable contingent claims in arbitrage theory, Ann. Inst. H. Poincaré. Probab. Statist. 33 (1997), 113-144.

Departement für Mathematik, ETH Zürich, CH-8092 Zürich, Switzerland

E-mail address: delbaen@math.ethz.ch

Institut für Statistik, Universität Wien, Brünnerstr. 72, A-1210 Wien, Austria

E-mail address: wschach@stat1.bwl.univie.ac.at 